Research Article

\title{
Stabilizing and Robust Fractional PID Controller Synthesis for Uncertain First-Order plus Time-Delay Systems
}

\author{
Aymen Rhouma $\mathbb{D}^{1},{ }^{1}$ Sami Hafsi $\mathbb{D}^{1},{ }^{1,2}$ and Kaouther Laabidi ${ }^{1}$ \\ ${ }^{1}$ University of Tunis Manar, National Engineering School of Tunis, LR11ES20 Analysis, \\ Conception and Control of Systems Laboratory, Tunis 1002, Tunisia \\ ${ }^{2}$ University of Kairouan, Higher Institute of Applied Sciences and Kairouan Technology, Kairouan, Tunisia \\ Correspondence should be addressed to Aymen Rhouma; aymenrh@yahoo.fr
}

Received 20 March 2021; Accepted 6 May 2021; Published 24 May 2021

Academic Editor: A. M. Nagy

Copyright (c) 2021 Aymen Rhouma et al. This is an open access article distributed under the Creative Commons Attribution License, which permits unrestricted use, distribution, and reproduction in any medium, provided the original work is properly cited.

\begin{abstract}
In this paper, by using the noninteger $P I^{\lambda} D^{\mu}$ controllers, we conduct an investigation into the subject of robust stability area of time-delay interval process. Our method is based on setting up of the noninteger interval closed-loop characteristic equation using the inferior and superior bounds of uncertain parameters into several vertexes. We have combined the composition of the value set of vertex with the zero exclusion principle to analyse the stability of the uncertain process. A generalized version of the Hermite-Biehler theorem, applicable to fractional quasipolynomials, is exploited to determine the stability region of each vertex. The robust stability region of the noninteger regulator can be given by the crossing of the stability area of all the vertex characteristic noninteger quasipolynomials. By using the value set method and zero exclusion theory, the effectiveness of the stability region can be tested. Also, we propose a suitable procedure to determine the whole of stabilizing parameters for an interval process. An explicative example is given to point out the advantage and reliability of the approach.
\end{abstract}

\section{Introduction}

The PID controllers are the best controllers applied in industrial applications due to their simplicity and their remarkable effectiveness of implementation [1-3]. Based on a little process knowledge, various simple and effective setting methods have been developed. As we know, control system is an active field of research, good results are forever required in order to achieve prescribed levels of performance. Meanwhile, alternative form PID regulators have been suggested to enhance the closed-loop response, for typical examples, IMC-PID [4-7] and Smith PID controller [8, 9].

The mathematical modeling and simulation of systems and processes based on the description of their physical laws leads to differential equations of a fractional order [10-12]. In fact, large efforts of academic and industrial research have been concentrated on modeling and fractional-order control, primarily in the fields of tuning techniques and stabilization approaches. The earliest contributions in this field are the three generations of CRONE controllers [13]. The authors of [13] proved the advanced performance of the CRONE regulators over the traditional PID controllers. Lately, Podlubny has suggested a new version of the PID regulator named fractional-order PID controller $\left(P I^{\lambda} D^{\mu}\right)$. This kind of controllers has received more and more attention. Thanks to the two extra freedom degree $\lambda$ and $\mu$, the $P I^{\lambda} D^{\mu}$ has showed a better response and a strong robustness, in correspondence with the standard PID, when applied for both integer-order and noninteger-order process $[14,15]$.

Many researchers find the key to the problem of stability, for example, Nyquist and Routh-Hurwitz stability criterions. Unfortunately, there are several problems to extend these results for noninteger systems. Recently, a few design approaches and tuning methods for $P I^{\lambda}, P D^{\mu}$, and $P I^{\lambda} D^{\mu}$ controllers have been presented from the generalizations of the classical rules. 
In [15], the authors designed a $P I^{\lambda}$ controller to fulfill three design specifications: robustness to system gain, phase margin variations, and time constant variations.

Also, an optimal noninteger-order regulator based on specified performances has been given in [16]. Many authors use the three stability boundaries, Real Root Boundary (RRB), Complex Root Boundary (CRB), and Infinite Root Boundary (IRB), to establish the stability area [17]. This approach yields the specific formula obverse to these limits in terms of noninteger-order controller parameters [18]. More recently, an effective method is proposed to compute the stability area of noninteger-order $P I^{\lambda} D^{\mu}$ for an arbitrarily given noninteger-order time-delay system [19].

For practical reasons, the authors of $[20,21]$ presented an implementation of the fractional-order integrator capable to realize a $P I^{\lambda} D^{\mu}$ controller.

We often face time delays in industrial systems and several problems in process control engineering. When dealing with a system involving time delays, controlled by a $P I^{\lambda} D^{\mu}$ controller, it is essential to be mindful that the closedloop characteristic equation is not a polynomial but a quasipolynomial function. The problem will be more complicated when the mathematical representation of the dynamic system suffers from uncertainties. These uncertainties are due to measurement fault, industrial tolerances, nonlinearities, and so on. Maybe the most famous result about interval systems is Kharitonov's theorem [22, 23]. This theorem proves that the interval polynomial set is Hurwitz stable if the four Kharitonov polynomials are Hurwitz stable.

The D-decomposition procedure has been widely employed to establish the stability region of fractional-order controllers manually, although this method presents some little complicated problems that still remain to be resolved $[17,18,24]$. For this method, a point in every area split by the boundaries with respect to the singular frequencies has to be picked to quantify the process stability in the sense of the Nyquist rule. If the number of the fragmented areas is big, the technique of computing stability region will be needless. Consequently, the stability region of the $P I^{\lambda} D^{\mu}$ controller in this case cannot be carried out by this method.

In this paper, an efficient procedure is proposed to compute the stability area of noninteger regulator of timedelay system. The motivation here is to resolve the case where $\lambda+\mu=2$, and this case cannot be achieved by other methods. The results also expose many forms of stabilizing areas for the $P I^{\lambda} D^{\mu}$ regulators. It should be noted that, for the uncertain first-order process with time delay, the stability study was not well elaborated and it is still a renewed research area. Indeed there are not various research works that deal with the problem of uncertain parameters of time-delay systems with noninteger PID regulators.

Motivated by the above discussion, a robust stabilization method of $P I^{\lambda} D^{\mu}$ regulators for first-order process with time delay is given in this work. The objective is to determine the stability area of the $P I^{\lambda} D^{\mu}$ controllers in the $\left(K_{p}, K_{i}, K_{d}\right)$ plane for the uncertain system.

This paper is organised as follows: in Section 2, we present Hermite-Biehler and Pontryagin theorems which will be useful to compute stability region of time-delay system. A method for the setting up of the value set is given and a new procedure is offered to handle the stability area of uncertain noninteger process in Section 3. In Section 4, an example of time-delay plant controlled by a noninteger regulator is given. Finally, Section 5 is reserved for the conclusion and some remarks.

\section{Stabilization of Uncertain First-Order Time- Delay System}

A SISO noninteger control plant is shown in Figure 1.

In Figure 1, $r$ is the reference input, $u$ is the control law, $y$ is the output signal, and $G(s)$ and $C(s)$ are the transfer functions, respectively, of the time-delay process and the noninteger PID controller.

Our objective in this work is to compute the set of $\left(K_{p}, K_{i}, K_{d}\right)$ parameters where the closed-loop process stays stable, where $K_{p}$ is the proportional gain, $K_{i}$ is the integral gain, and $K_{d}$ is the derivative gain, and the orders $\lambda$ and $\mu$ may assume real noninteger positives values. The output of the overall noninteger control process is given by

$$
y(s)=\frac{C(s) G(s)}{1+C(s) G(s)} r(s) .
$$

The complex closed-loop characteristic equation of this system is given as

$$
Q(j \omega)=q_{r}(\omega)+j q_{i}(\omega)
$$

where $q_{r}(\omega)$ and $q_{i}(\omega)$ expose the real and the imaginary part, respectively, of $Q(j \omega)$.

The stabilization of this kind of systems is relatively complicated, since the number of roots is infinite when time delays are introduced. In this work, we use the generalization Hermite-Biehler theorem for quasipolynomials [1, 25]. This theorem is useful to find the fractional-order PID regulator parameters that stabilize a given time-delay systems.

Hence, the characteristic equation of the closed-loop system is

$$
Q(s)=d(s)+e^{-s L_{i}} n_{i}(s)
$$

where $d(s)$ and $n_{i}(s)$ for $i=1,2, \ldots, m$ are quasipolynomials with real coefficients:

$$
\begin{aligned}
& \operatorname{deg}(d(s))=n, \\
& \operatorname{deg}\left(n_{i}(s)\right)<n, \quad \text { for } i=1,2, \ldots, m .
\end{aligned}
$$

$L_{i}$ for $i=1,2, \ldots, m$ represent time delays with order $L_{1}<L_{2}<\cdots<L_{m}$.

From equation (1), we can define the fractional characteristic expression of the process in Figure 1 as

$$
Q(s)=s^{\lambda}(1+T s)+K\left(K_{p} s^{\lambda}+K_{i}+K_{d} s^{\mu+\lambda}\right) e^{-L s} .
$$

We can rewrite the quasipolynomial $Q(s)$ as follows:

$$
Q(s)=s^{\lambda}(1+T s) e^{L s}+K\left(K_{p} s^{\lambda}+K_{i}+K_{d} s^{\mu+\lambda}\right) .
$$

In order to guarantee that $Q(s)$ admits a nonzero principal term, for this, it is necessary that the coefficients of 


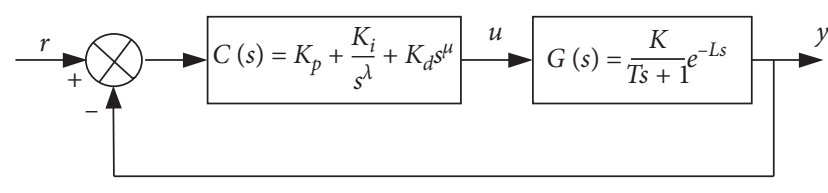

Figure 1: Closed-loop noninteger system.

the higher degrees of $s$ and $e^{s}$ are different from zero, we must suppose that $\operatorname{deg}(d(s))=\lambda+1>\operatorname{deg}(n(s))=\lambda+\mu$. This assumption implies that $\mu<1$; in this work, we suppose that $\lambda+\mu=2$, where $\lambda=(2 / 3)$ and $\mu=(1 / 3)$.

Then, for $z=L s, Q(s)$ becomes

$$
Q(z)=\left(\frac{z}{L}\right)^{\lambda}\left(1+\frac{T}{L} z\right) e^{z}+K\left(K_{p}\left(\frac{z}{L}\right)^{\lambda}+K_{i}+K_{d}\left(\frac{z}{L}\right)^{\mu+\lambda}\right) \text {. }
$$

By replacing $z$ by $j \omega$, the noninteger quasipolynomial (7) is given by

$$
\begin{aligned}
Q(j \omega)= & \left(\frac{j \omega}{L}\right)^{\lambda}\left(1+\frac{T}{L} j \omega\right) e^{j \omega}+K K_{p}\left(\frac{j \omega}{L}\right)^{\lambda}+K K_{i} \\
& +K K_{d}\left(\frac{j \omega}{L}\right)^{\mu+\lambda} .
\end{aligned}
$$

\subsection{Determination of $K_{p}$ Range}

2.1.1. Hermite-Biehler Theorem $[1,25]$ Let $Q(j \omega)$ be in the following form:

$$
Q(j \omega)=q_{r}(\omega)+j q_{i}(\omega),
$$

where $q_{r}(\omega)$ and $q_{i}(\omega)$, respectively, represent the real and the imaginary part of $Q(j \omega)$.

$Q(j \omega)$ is stable if and only if

(1) $q_{r}(\omega)$ and $q_{i}(\omega)$ have only simple real roots and these are interlaced.

(2) $q_{i}^{\prime}(\omega) q_{r}(\omega)-q_{i}(\omega) q_{r}^{\prime}(\omega)>0, \quad$ for $\quad$ some $\omega \in]-\infty ;+\infty[$,

where $q_{r}^{\prime}(\omega)$ and $q_{i}^{\prime}(\omega)$, respectively, represent the derivatives of $q_{r}(\omega)$ and $q(\omega)$.

A key step in Hermite-Biehler theorem to examine stability of the process is to assure that $q_{r}(\omega)$ and $q_{i}(\omega)$ have just real roots. This characteristic can be tested due to Pontryagin theorem $[26,27]$.

2.1.2. Pontryagin Theorem. $M$ and $N$ indicate the uppermost degrees of $s$ and $e^{s}$, respectively, in $Q(j \omega)$, and $\eta$ is a suit constant such that the coefficients of terms of uppermost degree in $q_{r}(\omega)$ and $q_{i}(\omega)$ do not become zero at $\omega=\eta$. To fulfill that the equations $q_{r}(\omega)=0$ and $q_{i}(\omega)=0$ have just real roots, it is necessary and sufficient that

$$
-2 l \pi+\eta \leq \omega \leq 2 l \pi+\eta ; \quad l=1,2,3, \ldots .
$$

$q_{r}(\omega)$ and $q_{i}(\omega)$ have exactly $4 l N+M$ roots, with large $l . \diamond$ This theorem allows us to specify the suitable value of $\eta$ so as to get the widest interval of $K_{p}$ parameter to make sure that $q_{r}(\omega)$ and $q_{i}(\omega)$ have just real roots. According to $\mathrm{H}$. B theorem, it is useless to verify if roots of the imaginary and real parts are all real. Since the interlacing ownership between the roots of $q_{r}(\omega)$ and $q_{i}(\omega)$ is confirmed, the other part has also just only real roots.

For $(j \omega)^{\lambda}=\omega^{\lambda}(\cos ((\pi / 2) \lambda)+j \sin ((\pi / 2) \lambda)), q_{r}(\omega)$ and $q_{i}(\omega)$ can be expressed by

$$
\begin{aligned}
q_{r}(\omega)= & \left(\frac{\omega}{L}\right)^{\lambda} \cos \left(\frac{\pi}{2} \lambda\right)\left(K K_{p}+\cos (\omega)-\frac{T}{L} \omega \sin (\omega)\right) \\
& -\left(\frac{\omega}{L}\right)^{\lambda} \sin \left(\frac{\pi}{2} \lambda\right)\left(\sin (\omega)+\frac{T}{L} \omega \cos (\omega)\right)+K K_{i} \\
& +K K_{d}\left(\frac{\omega}{L}\right)^{\mu+\lambda} \cos \left(\frac{\pi}{2}(\lambda+\mu)\right),
\end{aligned}
$$

$$
\begin{aligned}
q_{i}(\omega)= & \left(\frac{\omega}{L}\right)^{\lambda} \cos \left(\frac{\pi}{2} \lambda\right)\left(\sin (\omega)+\frac{T}{L} \omega \cos (\omega)\right)+\left(\frac{\omega}{L}\right)^{\lambda} \\
& \sin \left(\frac{\pi}{2} \lambda\right)\left(K K_{p}+\cos (\omega)-\frac{T}{L} \omega \sin (\omega)\right) \\
& +K K_{d}\left(\frac{\omega}{L}\right)^{\mu+\lambda} \sin \left(\frac{\pi}{2}(\lambda+\mu)\right) .
\end{aligned}
$$

Clearly, the $P I^{\lambda} D^{\mu}$ gains $K_{p}$ and $K_{d}$ appear in the imaginary part (12), while the real part (11) is affected by all controller parameters. In order to examine the stability of closed-loop system, we require to compute all controller parameter $\left(K_{p}, K_{i}, K_{d}\right)$. As a first step, based on P. theorem, we compute the range of the proportional gain $K_{p}$ which assures real roots for $q_{i}(\omega)$ with an appropriate $\eta$ value as explained in the previous theorem. In [28], the authors proposed an important method to carry out the stability area for the case $\lambda+\mu=2$, which cannot be determined by an other method for the stabilization of the noninteger-order $P I^{\lambda} D^{\mu}$ regulator. In such a case, it should be noted that we have cited this logical exigency $\lambda+\mu=2$ on the stabilizing noninteger-order regulator which is well defined due to the above assumption: $\operatorname{deg}(d(s))=\lambda+1>\operatorname{deg}(n(s))=\lambda+\mu$. As a consequence of this requirement, the term $\sin ((\pi / 2)(\lambda+\mu))$ is equal to zero and the imaginary part is as follows:

$$
\begin{aligned}
q_{i}(\omega)= & \left(\frac{\omega}{L}\right)^{\lambda} \cos \left(\frac{\pi}{2} \lambda\right)\left(\sin (\omega)+\frac{T}{L} \omega \cos (\omega)\right)+\left(\frac{\omega}{L}\right)^{\lambda} \\
& \sin \left(\frac{\pi}{2} \lambda\right)\left(K K_{p}+\cos (\omega)-\frac{T}{L} \omega \sin (\omega)\right) .
\end{aligned}
$$

It is remarkable to note that function (13) depends on only one unknown fractional-order PID parameter. Therefore, as stated by H. B theorem, we use the P. theorem to determine the range of $K_{p}$ parameter which ensures that the real and imaginary parts have only real roots for a given range of $\eta$.where $N_{r}$ represents the number of roots for $q_{i}^{*}(\omega)$ and $K_{p 1}$ and $K_{p 2}$ represent the first interval limits of 
$K_{p}$ arbitrary chooses. The values of $K_{p}$ ensuring real roots for $q_{i}(\omega)$ are given by Algorithm 1, as shown in Figure 2. It is possible to note that the projection $\eta=0.8$ corresponds to the largest range for $K_{p}$.

From this figure, we choose an appropriate value of $\eta=$ 1.9 which fulfills the condition that $\eta$ would not vanish both real and imaginary parts; this value corresponds to $-0.9<K_{p}<1.6$. After determining the $K_{p}$ range, we present an elegant procedure in order to compute the set $\left(K_{i}, K_{d}\right)$ values.

2.2. Stability Area in $\left(K_{i}, K_{d}\right)$ Plan. In this section, we compute the whole $K_{i}$ and $K_{d}$ gains of the $P I^{\lambda} D^{\mu}$ regulator that make system stable. In fact, by taking into account the interlacing property between the roots of the two parts of $Q(j \omega)$ and using the lemma given in [29], we calculate $q_{r}(\omega)$ with the zeros of $q_{i}(\omega)$.

Lemma 1. For $K_{p}$ range given by Algorithm 1 , the property of interlacing between the roots of $q_{i}(z)$ and $q_{r}(z)$ is handled by

$$
\begin{aligned}
& (-1)^{j} q_{r}\left(z_{j}\right)>0 \text {, } \\
& j=0,1,2, \ldots \\
& \left\{\begin{array}{l}
q_{r}\left(z_{0}\right)>0 \\
q_{r}\left(z_{1}\right)<0 \\
q_{r}\left(z_{2}\right)>0 \longrightarrow \\
q_{r}\left(z_{3}\right)<0 \\
\vdots \\
\pm K_{d}<-\left(\frac{L}{z_{1}}\right)^{\mu}\left(B\left(z_{1}\right)+m\left(z_{1}\right) K_{i}\right) \\
\pm K_{d}>-\left(\frac{L}{z_{2}}\right)^{\mu}\left(B\left(z_{2}\right)+m\left(z_{2}\right) K_{i}\right), \\
\pm K_{d}<-\left(\frac{L}{z_{3}}\right)^{\mu}\left(B\left(z_{3}\right)+m\left(z_{3}\right) K_{i}\right) \\
\vdots
\end{array}\right.
\end{aligned}
$$

where

$$
\begin{aligned}
B\left(z_{j}\right)= & \cos \left(\frac{\pi}{2} \lambda\right)\left(K_{p}+\frac{1}{k}\left(\cos \left(z_{j}\right)-\frac{T}{L} z_{j} \sin \left(z_{j}\right)\right)\right) \\
& -\frac{1}{K} \sin \left(\frac{\pi}{2} \lambda\right)\left(\sin \left(z_{j}\right)+\frac{T}{L} z_{j} \cos \left(z_{j}\right)\right), \\
m\left(z_{j}\right)= & \left(\frac{L}{z_{j}}\right)^{\lambda} .
\end{aligned}
$$

The borderlines of these areas are shown in Figure 3, which are determined by straight lines with the following equations:

$$
K_{d}=-\left(\frac{L}{\omega_{j}}\right)^{\mu}\left(B\left(\omega_{j}\right)+m\left(\omega_{j}\right) K_{i}\right) .
$$

The shaded area of these four lines gives the stability region in the $\left(K_{i}, K_{d}\right)$ plane with $K_{p}=0.4$.

In view of the above lemma, we show this second algorithm to calculate the ensemble of stabilizing $\left(K_{i}, K_{d}\right)$ parameters for a fixed $K_{p}$ parameter. (Algorithm 2)

\section{Generalisation of the Hermite-Biehler Criterion Stability for Uncertain Noninteger- Order Quasipolynomial}

We exploit the studies presented previously to discuss the stability of the uncertain noninteger-order time-delay systems. For that, let us consider the uncertain first-order system as follows:

$$
G(s)=\frac{K}{1+T s} e^{-L s},
$$

where the intervals of parameters are

$$
\begin{aligned}
& K \in[\underline{K} ; \bar{K}], \\
& T \in[\underline{T} ; \bar{T}], \\
& L \in[\underline{L} ; \bar{L}] .
\end{aligned}
$$

The objective of this article is to compute the robust stability region of the interval time-delay system using the noninteger-order $P I^{\lambda} D^{\mu}$ regulator. Based on the argument value interval concept, we construct the geometrical techniques of complex analysis. In fact, the uncertain fractional- 
(1) Initialize step $=0.1 ; N_{r}=0 ; K_{p}=K_{p 1}$.

(2) Count the roots of the equation $q_{i}(\omega)=0$ for $\omega \in[-2 \pi+\eta: 2 \pi+\eta]$.

(3) If $N_{r}=4 l N+M$ then go to 4 .

Else increment $K_{p}=K_{p}+$ step and go to 5 .

(4) Save $K_{p}$ and increment $K_{p}=K_{p}+$ step.

(5) If $K_{p}<K_{p 2}$, go to 2 , else give Pontryagin interval,

Algorithm 1: Compute $K_{p}$ range for several $\eta$ values.

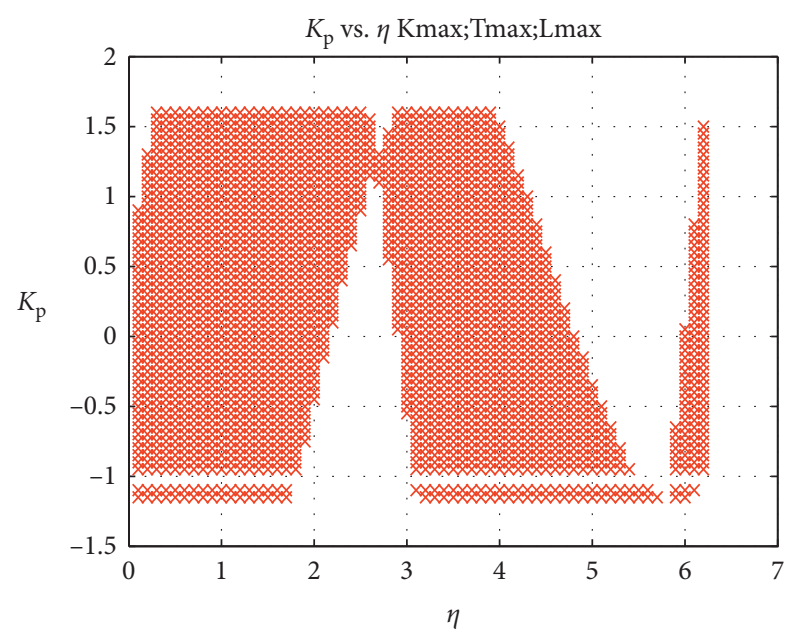

Figure 2: Different values of $K_{p}$ for $\eta \in[0: 6.2]$.

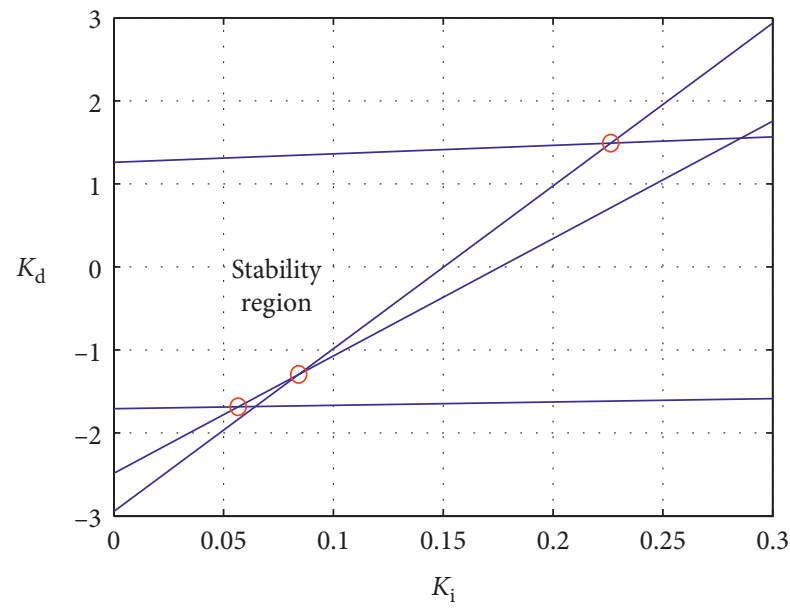

FIgURE 3: Stability region for $K_{p}=0.4$.

order first-order system is dissociated into diverse vertexes of uncertain parameters. Based on these vertexes of parameters, we construct the set of values of characteristic quasipolynomials. The method presented in Section 2 is used to determine the stability area of each vertex of the closedloop characteristic quasipolynomial. The stability region of the interval system is given by the intersection of these stability areas which is called robust stability region.

Using the lower and upper bounds, the interval timedelay system $G(s)$ given by equation (17) can be decomposed into $2^{3}=8$ vertex fractional-order quasipolynomials and
$3 * 2^{2}=12$ exposed edges. Therefore, by taking a point $\left(K_{i}, K_{d}\right)$ from the stability region in Figure 3, given for fixed $K_{p}=0.4$, we can write all the eight vertex fractional quasipolynomials of $Q(s, p)$ in the following pattern:

$$
\begin{aligned}
& q_{1}(s, p)=s^{\lambda}(1+\underline{T} s) e^{\underline{L} s}+\underline{K}\left(K_{p} s^{\lambda}+K_{i}+K_{d} s^{\mu+\lambda}\right), \\
& q_{2}(s, p)=s^{\lambda}(1+\bar{T} s) e^{\underline{L} s}+\underline{K}\left(K_{p} s^{\lambda}+K_{i}+K_{d} s^{\mu+\lambda}\right), \\
& q_{3}(s, p)=s^{\lambda}(1+\bar{T} s) e^{\underline{L} s}+\bar{K}\left(K_{p} s^{\lambda}+K_{i}+K_{d} s^{\mu+\lambda}\right), \\
& q_{4}(s, p)=s^{\lambda}(1+\underline{T} s) e^{\underline{L} s}+\bar{K}\left(K_{p} s^{\lambda}+K_{i}+K_{d} s^{\mu+\lambda}\right), \\
& q_{5}(s, p)=s^{\lambda}(1+\underline{T} s) e^{\bar{L} s}+\underline{K}\left(K_{p} s^{\lambda}+K_{i}+K_{d} s^{\mu+\lambda}\right), \\
& q_{6}(s, p)=s^{\lambda}(1+\bar{T} s) e^{\bar{L} s}+\underline{K}\left(K_{p} s^{\lambda}+K_{i}+K_{d} s^{\mu+\lambda}\right), \\
& q_{7}(s, p)=s^{\lambda}(1+\bar{T} s) e^{\bar{L} s}+\bar{K}\left(K_{p} s^{\lambda}+K_{i}+K_{d} s^{\mu+\lambda}\right), \\
& q_{8}(s, p)=s^{\lambda}(1+\underline{T} s) e^{\bar{L} s}+\bar{K}\left(K_{p} s^{\lambda}+K_{i}+K_{d} s^{\mu+\lambda}\right) .
\end{aligned}
$$

For example, we remark from equation (19) that $q_{1}(s, p)$ and $q_{2}(s, p)$ have the same structure except the value of the parameter $T$. Hence, one of the exposed edges can be presented by the following expression:

$$
(1-\tau) q_{1}(s)+\tau q_{2}(s), \tau=[0,1] .
$$

In the same way, we build the other exposed edges and we determine the ensemble which contains $3 * 2^{2}=12$ exposed edges as

$$
\begin{aligned}
Q_{E}(s)= & \left\{e\left(q_{1}(s), q_{2}(s)\right), e\left(q_{1}(s), q_{3}(s)\right), e\left(q_{1}(s), q_{5}(s)\right),\right. \\
& e\left(q_{2}(s), q_{4}(s)\right), e\left(q_{2}(s), q_{8}(s)\right), e\left(q_{3}(s), q_{7}(s)\right), \\
& e\left(q_{3}(s), q_{4}(s)\right), e\left(q_{4}(s), q_{8}(s)\right), e\left(q_{5}(s), q_{6}(s)\right), \\
& \left.e\left(q_{5}(s), q_{7}(s)\right), e\left(q_{6}(s), q_{8}(s)\right), e\left(q_{7}(s), q_{8}(s)\right)\right\} .
\end{aligned}
$$

For further survey, the lector can refer to [30] and to the extensive results on such fractional uncertain quasipolynomials given in [26]. In this context, we give the following theorems.

Theorem 1 (see $[29,31]$ ). The uncertain parameters are linearly dependent to each other for single delay systems and fixed \$lomega\$. Then, by mapping the vertices $\$ \mathrm{q} i \mathrm{i}(\mathrm{s}$, q) Sand the exposed edges $\$ Q \_E\left(s^{*}\right) \$$, we obtain a convex polygon in the complex plane (Figure 4 ). From the complex 
(1) Compute $K_{p}$ interval using Algorithm 1.

(2) Select a $K_{p}$ value from the already determined range.

(3) Get the roots $\omega_{j}$ from equation (11).

(4) Compute the palrameters $B\left(\omega_{j}\right)_{j=1 \ldots 4}$ and $m\left(\omega_{j}\right)_{j=1 \ldots 4}$.

(5) Delimit the $\left(K_{i}, K_{d}\right)$ area using equation (16).

(6) If $K_{p}<K_{p 2}$ return to Step 2.

Algorithm 2: Stability region for a system with fixed parameters.

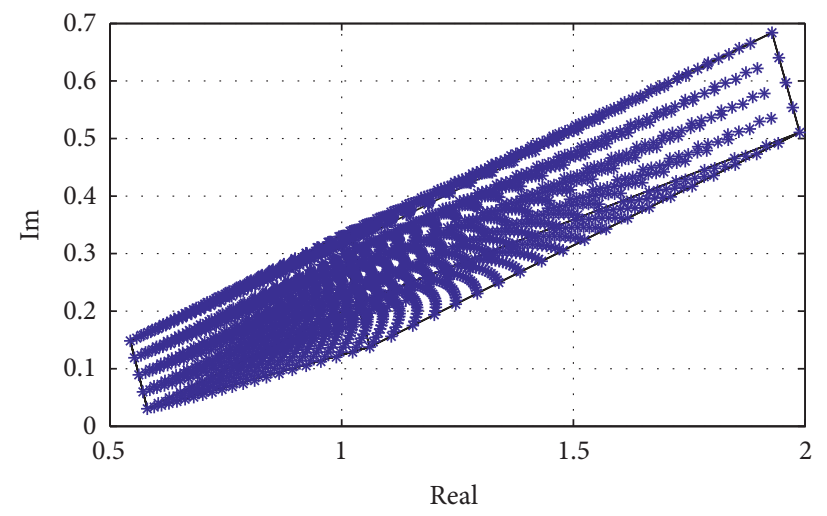

FIgURE 4: The images of 2205 quasipolynomials and the exposed edges of equation (19).

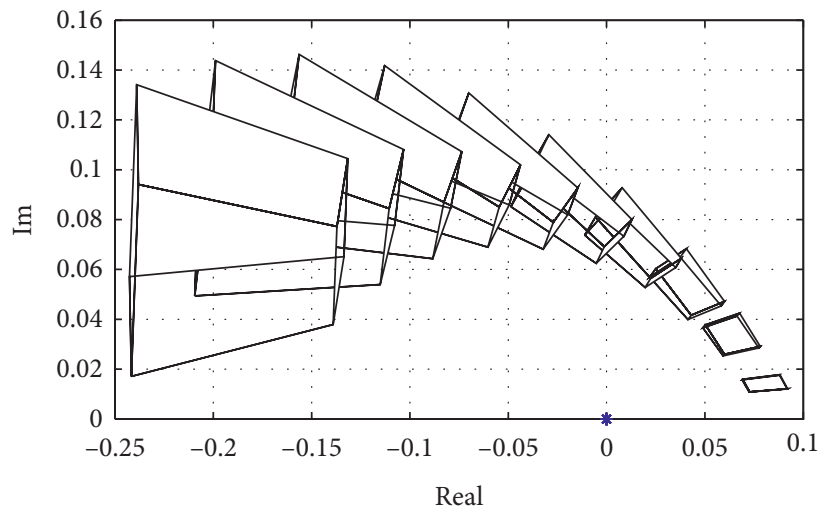

Figure 5: The value sets of interval polynomial of equation (19) for $0 \leq \omega \leq 4$.

plane geometry, one can say that the boundary of the value set of the fractional quasipolynomial $\$ Q\left(j \backslash\right.$ omega* ${ }^{*}$ q)\$ can be obtained from the images of the exposed edges

$$
\partial Q\left(s^{*}, q\right) \subset Q_{E}\left(s^{*}\right),
$$

where $\partial$ indicates the limit and $Q_{E}$ is given in (21)

Theorem 2 (see [29]). The noninteger-order uncertain quasipolynomial $Q\left(j \omega^{*}, q\right)$ is robust stable if $Q_{E}\left(j \omega^{*}\right)$ is stable.
In this work, we choose $T=[1.5,2.5], L=[3,4]$, and $K=$ $[0.8,1.2]$ and we depict the value sets $Q_{E}(j \omega, p)$. Therefore, by taking 21 points within the uncertain parameters $T=$ [1.5: 0.05: 2.5] and $L=$ [1: 0.05: 2] and 5 points within the uncertain parameter $K=[0.8: 0.05: 1.2]$, the images of $21 \times 21 \times 5=2205$ quasipolynomials at $\omega=4 \mathrm{rad} / \mathrm{s}$ are shown in Figure 4. Also, the images and the exposed edges at $\omega=4 \mathrm{rad} / \mathrm{s}$ are shown in the same figure by using a solid line. We can also see that the value set is limited by the set of exposed edges. 
(1) Define $Q\left(s ; K_{p}, K_{i}, K_{d}\right)$ by equation (19).

(2) Produce exposed edges by equation (20).

(3) Compose the set of exposed edges, $Q_{E}(s ; p)$, equation (21).

(4) Using Algorithm 1, the allowable ranges of $K_{p}$ of each vertex (19) is found.

(5) Determine the intersection of all the $K_{p}$ intervals.

(6) Using Algorithm 2, we compute the $\left(K_{i}, K_{d}\right)$ stabilizing values for the entire family.

(7) Plot the robust stability region for every vertex in the $\left(K_{p}, K_{i}, K_{d}\right)$ plan.

Algorithm 3: Stability of uncertain time-delay system.

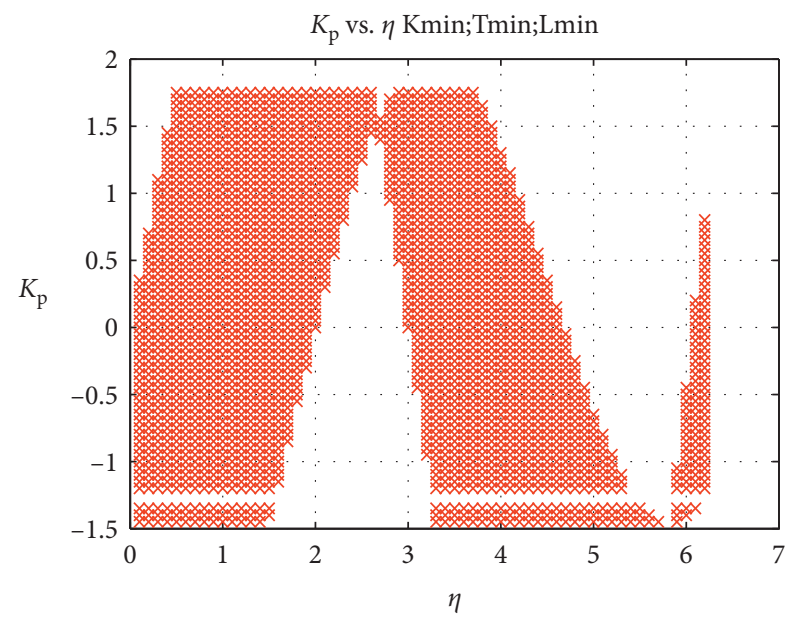

(a)

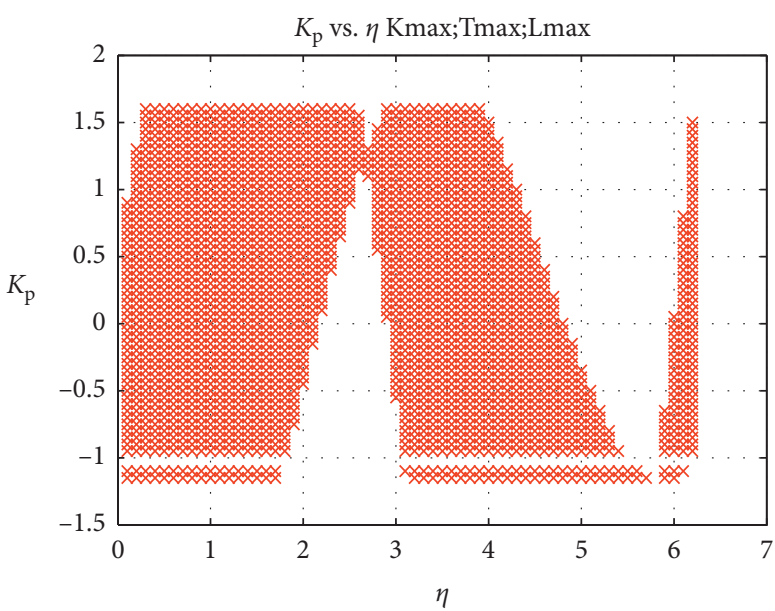

(b)

Figure 6: $K_{p}$ vs. $\eta$ as computed by applying Pontryagin theorem: (a) $(\underline{K}, \underline{T}, \underline{L})$; (b) $(\bar{K}, \bar{T}, \bar{L})$.

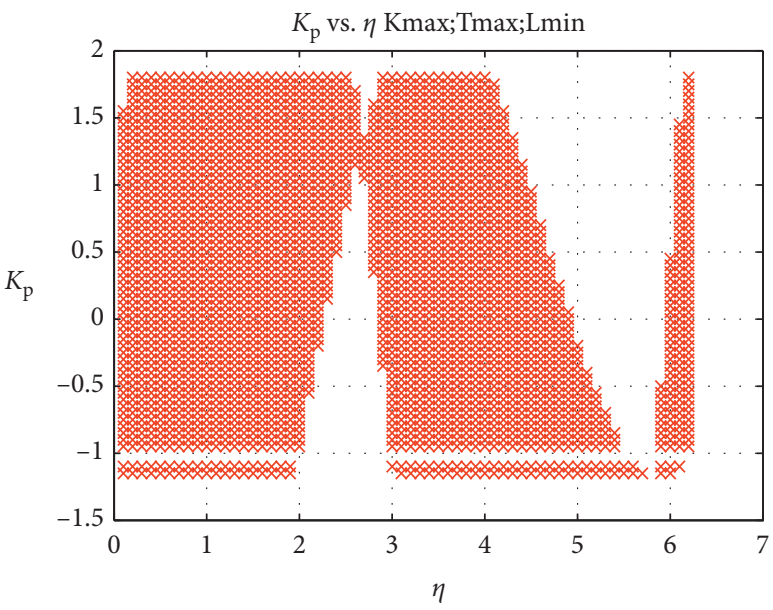

(a)

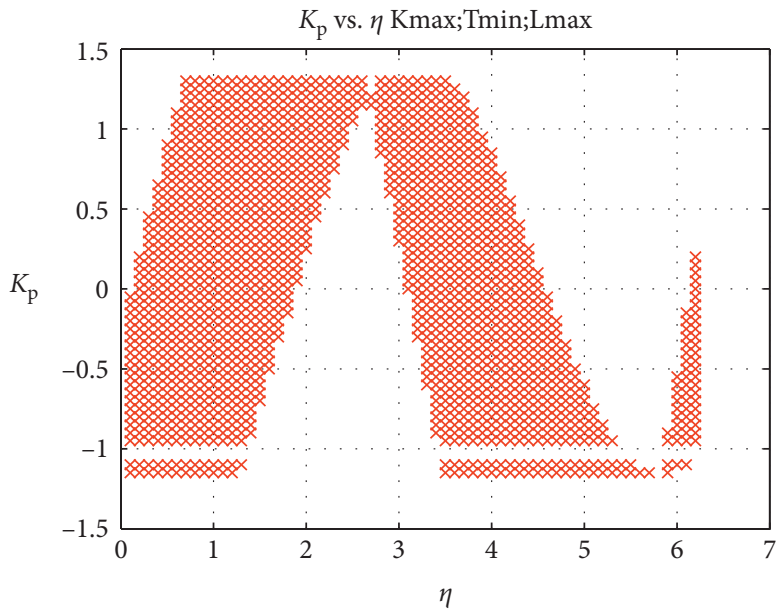

(b)

Figure 7: $K_{p}$ vs. $\eta$ as computed by applying Pontryagin theorem: $(\mathrm{a})(\bar{K}, \bar{T}, \underline{L}) ;(\mathrm{b})(\bar{K}, \underline{T}, \bar{L})$. 


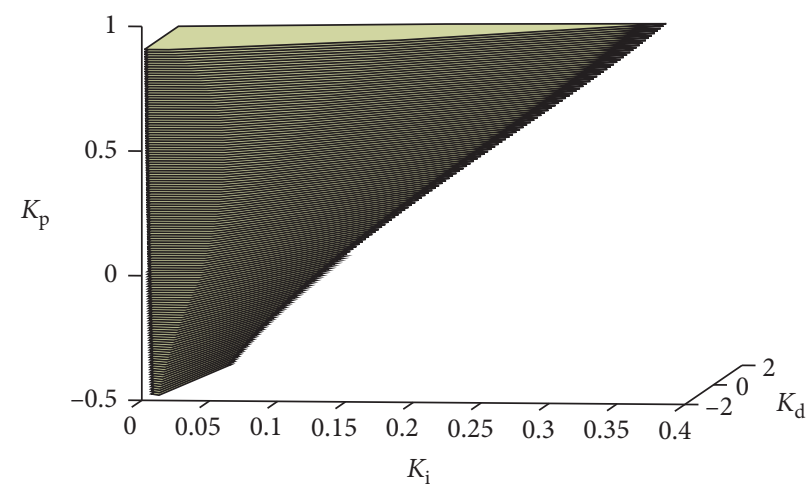

Figure 8: The global stability region for the $P I^{2 / 3} D^{1 / 3}$ controller.

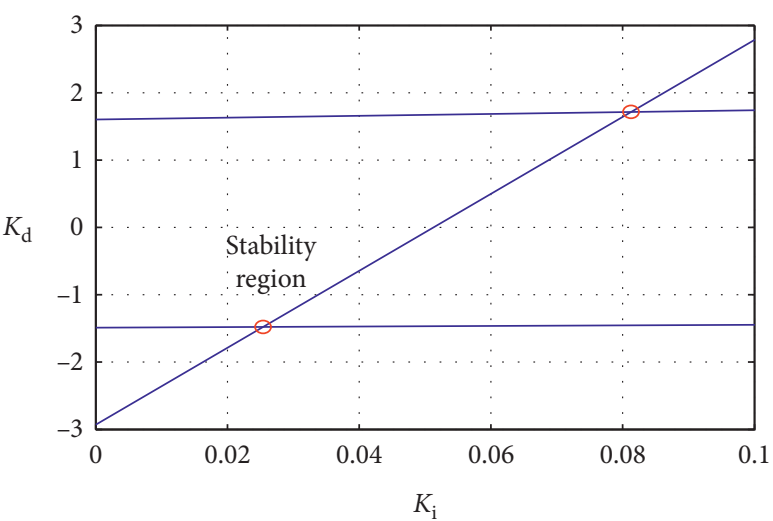

(a)

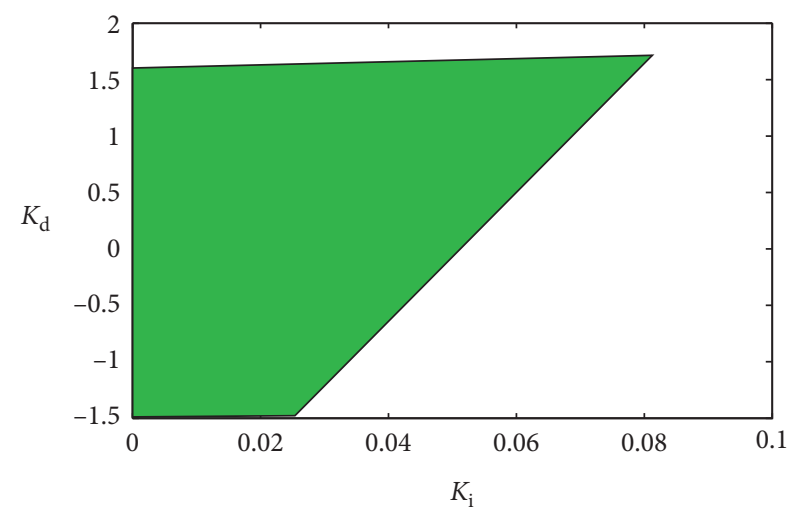

(b)

Figure 9: (a) The boundary lines of the stabilizing set in the $\left(K_{i}, K_{d}\right)$ space for $K_{p}=-0.1$. (b) Stability region in the $\left(K_{i}\right.$, $\left.K_{d}\right)$ plane for $K_{p}=-0.1$.

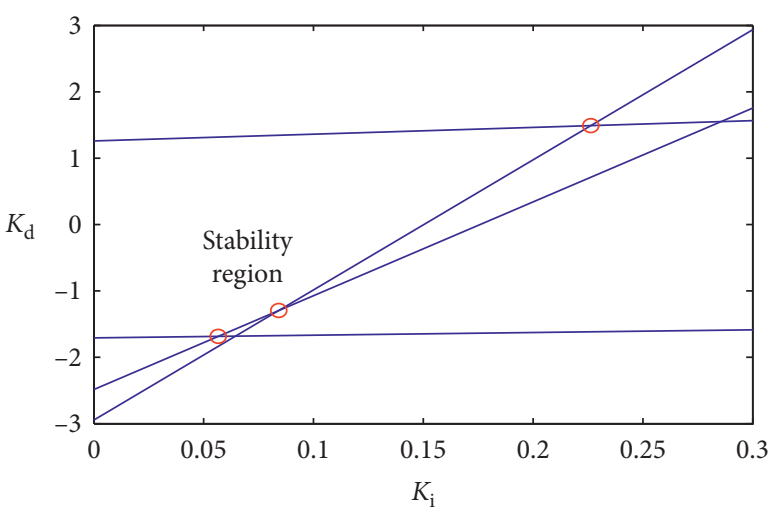

(a)

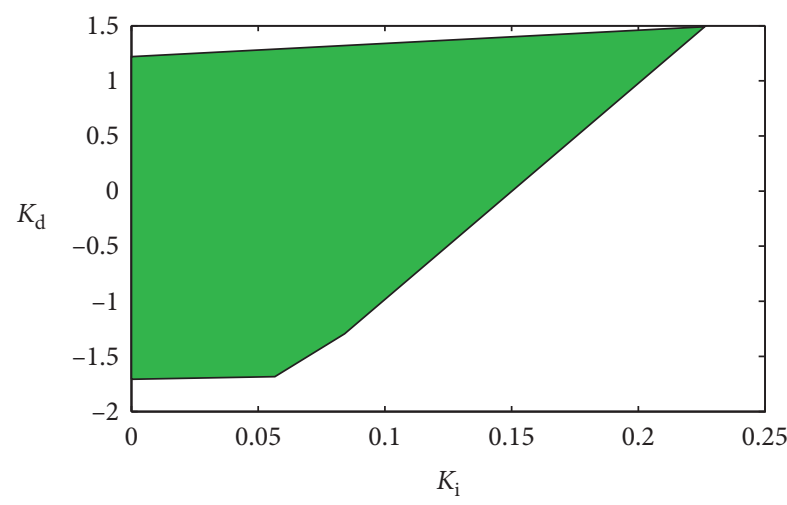

(b)

Figure 10: (a) The boundaries lines of the stabilizing set in the $\left(K_{i}, K_{d}\right)$ space for $K_{p}=0.4$. (b) Stability region in the $\left(K_{i}, K_{d}\right)$ plane for $K_{p}=0.4$. 


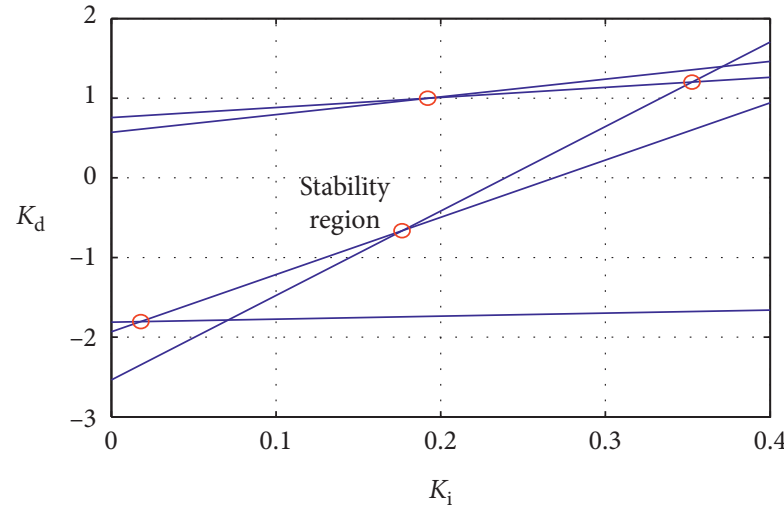

(a)

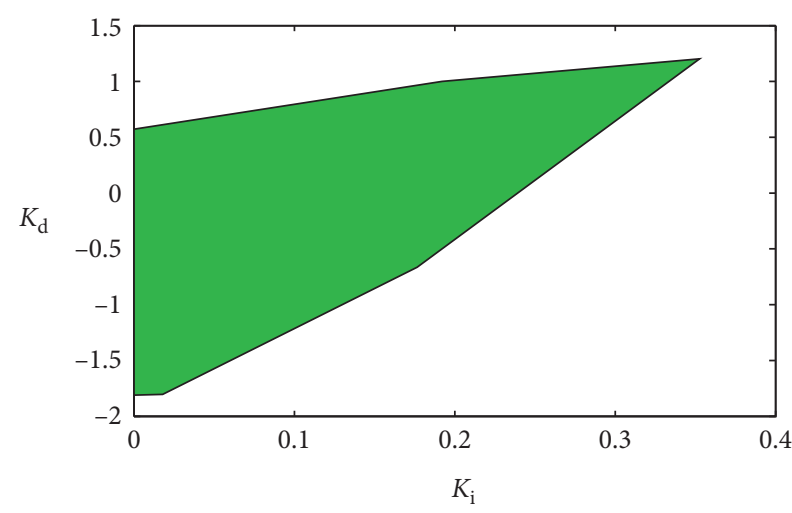

(b)

Figure 11: (a) The boundaries lines of the stabilizing set in the $\left(K_{i}, K_{d}\right)$ space for $K_{p}=0.9$. (b) Stability region in the $\left(K_{i}, K_{d}\right)$ plane for $K_{p}=0.9$.

Theorem 3 (see [29]). $Q(s, q)$ is a stable quasipolynomial family if there exists at least one point $s^{* 0} \in \partial Q\left(s^{*}, q\right)$ such that $0 \notin Q\left(s^{* 0}, q\right)$ for all frequencies.

According to Theorem 2, it is possible to ensure the robust stability of the uncertain quasipolynomials given in (19). Consequently, we can ensure that the entire fractional quasipolynomial family $\mathrm{Q}\left(s ; K_{p}, K_{i}, K_{d}\right)$ is a stable fractional quasipolynomial family if it has at least one stable vertex fractional quasipolynomial $Q_{j}\left(s ; K_{p}, K_{i}, K_{d}\right)$ and the value set of $Q_{E}(s, p)$ does not include the origin for all frequencies.

It is clear from Figure 5 that the origin is not included in the value sets for $0 \leq \omega \leq 4$. Therefore, based on Theorem 3 , the fractional-order controlled system is robust stable. Let us now summarize the simple and efficient Algorithm 3.

\section{Illustrate Example}

We consider the time-delay first-order system, where $K=[0.8,1.2], T=[1.5: 2.5]$, and $L=[1: 2]$. Based on the closed-loop characteristic equation given from expression (1), we determine the vertex fractional quasipolynomials as follows:

$$
\begin{aligned}
& q_{1}(s, p)=s^{\lambda}(1+1.5 s) e^{s}+0.8\left(K_{p} s^{\lambda}+K_{i}+K_{d} s^{\mu+\lambda}\right), \\
& q_{2}(s, p)=s^{\lambda}(1+2.5 s) e^{s}+0.8\left(K_{p} s^{\lambda}+K_{i}+K_{d} s^{\mu+\lambda}\right), \\
& q_{3}(s, p)=s^{\lambda}(1+2.5 s) e^{s}+1.2\left(K_{p} s^{\lambda}+K_{i}+K_{d} s^{\mu+\lambda}\right), \\
& q_{4}(s, p)=s^{\lambda}(1+1.5 s) e^{s}+1.2\left(K_{p} s^{\lambda}+K_{i}+K_{d} s^{\mu+\lambda}\right), \\
& q_{5}(s, p)=s^{\lambda}(1+1.5 s) e^{2 s}+0.8\left(K_{p} s^{\lambda}+K_{i}+K_{d} s^{\mu+\lambda}\right), \\
& q_{6}(s, p)=s^{\lambda}(1+2.5 s) e^{2 s}+0.8\left(K_{p} s^{\lambda}+K_{i}+K_{d} s^{\mu+\lambda}\right), \\
& q_{7}(s, p)=s^{\lambda}(1+2.5 s) e^{2 s}+1.2\left(K_{p} s^{\lambda}+K_{i}+K_{d} s^{\mu+\lambda}\right), \\
& q_{8}(s, p)=s^{\lambda}(1+1.5 s) e^{2 s}+1.2\left(K_{p} s^{\lambda}+K_{i}+K_{d} s^{\mu+\lambda}\right) .
\end{aligned}
$$

The main idea is to discuss the stability of each closedloop vertex characteristic quasipolynomial. The intersection stability area gives the robust stability area of the fractional- order interval plant. By applying the first algorithm to every vertex fractional quasipolynomials in equation (23), we obtain the $K_{p}$ ranges for each $\eta$ value as shown in Figures 6(a), 6(b), 7(a), and 7(b).

For $\eta=0.9, K_{p}=[-0.9: 1.7]$ is the intersection range of the eight $K_{p}$ ranges given for each $q_{j=1 \ldots 8}(s, p)$. For each value of $K_{p}$ inside this common region, the imaginary part $q_{i}(\omega)$ has five roots in $-2 \pi+\eta \leq \omega \leq 2 \pi+\eta$. Outside this nonempty intersection range of $K_{p}$, there are no stabilizing $P I^{4 / 3} D^{2 / 3}$ controllers.

By scanning over all $K_{p} \in(-0.9,1.7)$, we can yield the ensemble of $\left(K_{p}, K_{i}, K_{d}\right)$ values that make the closed-loop system stable. The robust stability region can be visualized in the three-dimensional plot $\left(K_{p}, K_{i}, K_{d}\right)$ for $\lambda=2 / 3$ and $\mu=1 / 3$, as shown in Figure 8. It is clear that the set of linear inequalities in terms of $\left(K_{i}, K_{d}\right)$ and this admissible set values have a special structure for each proportional gain, for example,

(i) For $K_{p}=-0.1$, the intersection area of the stability regions in the $\left(K_{i}, K_{d}\right)$ plane is a trapezoid as shown in Figures 9(a) and 9(b)

(ii) For $K_{p}=0.4$, the intersection area of the stability regions in the $\left(K_{i}, K_{d}\right)$ plane is a quadrilateral as shown in Figures 10(a) and 10(a)

(iii) For $K_{p}=0.9$, the intersection area of the stability regions in the $\left(K_{i}, K_{d}\right)$ plane is a polygon as shown in Figures 11(a) and 11(b).

\section{Conclusion}

A new procedure based on generalisation version of Hermite-Biehler theorem and Pontryagin theorem has been presented in this work to compute the robust stability region of a $P I^{\lambda} D^{\mu}$ regulator for an interval system with time delay. Using the lower and upper limits, the system with coefficient interval uncertainties is decomposed to various vertex plants. The stability area of each vertex characteristic quasipolynomial is given by the three algorithms which are based on Hermite-Biehler and Pontryagin theorems and zero exclusion principle. The common stability region of all vertex characteristic quasipolynomial defines the robust stability region. 


\section{Data Availability}

No data were used to support this study.

\section{Conflicts of Interest}

The authors declare that they have no conflicts of interest.

\section{References}

[1] G. J. Silva, A. Datta, and S. P. Bhattacharyya, PID Controllers for Time Delay Systems, Springer, London, UK, 2005.

[2] C. A. Monje, Q. Chen, B. M. Vinagre, X. Dingyu, and F. Vicente, Fractional-order Systems and Controls: Fundamentals and Applications (Advances in Industrial Control), Springer, London, UK, 2010.

[3] J. A. Karl and H. Tore, PID Controllers: Theory, Design and Tuning, Instrument Society of America, Research Traingle, NC, USA, 1995.

[4] I. C. Lung, "IMC-PID Controller Design," IFAC Adaptive Control of Chemical Processes, IFAC Proceedings, vol. 21, no. 7, pp. 147-152, Elsevier, Amsterdam, Netherlands, 1988.

[5] M. Shamsuzzoha and M. Lee, "IMC-PID controller design for improved disturbance rejection of time-delayed processes," Industrial \& Engineering Chemistry Research, vol. 46, no. 7, pp. 2077-2091, 2007.

[6] R. Ranganayakulu, G. Uday Bhaskar Babu, and A. Seshagiri, "Fractional filter IMC-PID controller design for second order plus time delay processes," Cogent Engineering, vol. 4, pp. 117, 2017.

[7] A. Leva and A. Colombo, "On the IMC-based synthesis of the feedback block of ISA-PID regulators," Transactions of the Institute of Measurement and Control, vol. 26, no. 5, pp. 417-440, 2004.

[8] K. J. Astrom, C. C. Hang, and B. C. Lim, "A new Smith predictor for controlling a process with an integrator and long dead-time," IEEE Transactions on Automatic Control, vol. 39, no. 2, pp. 343-345, 1994.

[9] J. Smith, "A controller to overcome dead time," ISA Journal, vol. 6, no. 2, pp. 28-33, 1959.

[10] J. A. Tenreiro Machado, "Analysis and design of fractionalorder digital control systems, systems analysis-modellingsimulation," Gordon and Breach Science Publishers, vol. 27, no. 3, pp. 107-122, 1997.

[11] R. Aymen, H. Sami, and L. Kaouther, "Fractional PI stabilization of delay systems: application to a thermal system," Journal of Applied Nonlinear Dynamics, vol. 8, no. 3, pp. 509-518, 2019.

[12] R. Aymen, H. Sami, and B. Faouzi, "Practical application of fractional order controllers to a delay thermal system," Computer Systems Science and Engineering, vol. 5, pp. 305313, 2019.

[13] A. Oustaloup, La Commande Crone, Hermès, New Castle, PA, USA, 1991.

[14] I. Podlubny, "Fractional-order systems and PI/sup/spl lambda//D/sup/spl mu//-controllers," IEEE Transactions on Automatic Control, vol. 44, no. 1, pp. 208-214, 1999.

[15] C. A. Monje, B. M. Vinagre, Y. Q. Chen, V. Feliu, P. Lanusse, and J. Sabatier, "Proposals for fractional $P I^{\lambda} D^{\mu}$ tuning," in Proceedings of the 1st IFAC Congress on Fractional Differentiation and its Applications, pp. 156-161, Bordeaux, France, July 2004.
[16] J. F. Leu, S. Y. Tsay, and C. Hwang, "Design of optimal fractional-order PID controllers," Chinese Journal of Chemical Engineering, vol. 33, no. 2, pp. 193-202, 2002.

[17] L. Taonian, C. Jianjun, and L. Chuang, "Algorithm of robust stability region for interval plant with time delay using fractional order $P I^{\lambda} D^{\mu}$ controller," Communications in Nonlinear Science and Numerical Simulation, vol. 17, pp. 979-991, 2012.

[18] S. E. Hamamci and M. Koksal, "Calculation of all stabilizing fractional-order PD controllers for integrating time delay systems," Computers \& Mathematics with Applications, vol. 59, no. 5, pp. 1621-1629, 2010.

[19] R. Caponetto, G. Dongola, L. Fortuna, and A. Gallo, "New results on the synthesis of FO-PID controllers," Communications in Nonlinear Science and Numerical Simulation, vol. 15, no. 4, pp. 997-1007, 2010.

[20] R. Caponetto and G. Dongola, "Field programmable analog array implementation of non integer order $P I^{\lambda} D^{\mu}$ controller," Journal of Computational and Nonlinear Dynamics, vol. 3, pp. 21-32, 2008.

[21] R. Caponetto and G. Dongola, "Analog implementation of non integer order $P I^{\lambda} D^{\mu}$ controller via field programmable analog array," in Proceedings of ASME International Design Engineering Technical Conferences (IDETC'07), Las Vegas, NV, USA, May 2007.

[22] V. L. Kharitonov, "The asymptotic stability of the equilibrium state of a family of systems of linear differential equations," Differentsialnye Uravneniya, vol. 14, pp. 2086-2088, 1978.

[23] S. P. Bhattacharyya, H. Chapellat, and L. H. Keel, Robust Control: The Parametric Approach, Prentice-Hall, Hoboken, NJ, USA, 1995.

[24] S. Zheng, X. Tang, and B. Song, "Graphical tuning method of FOPID controllers for fractional order uncertain system achieving robust D-stability," International Journal of Robust and Nonlinear Control, vol. 26, no. 5, pp. 1112-1142, 2016.

[25] R. Farkh, K. Laabidi, and M. Ksouri, "Computation of all stabilizing PID gain for second-order delay system," Mathematical Problems in Engineering, vol. 2009, Article ID 212053, 17 pages, 2009.

[26] S. Hafsi, L. Kaouther, and R. Farkh, "Synthesis of fractional PI controller for a first-order time delay system," Transactions of the Institute of Measurement and Control, vol. 35, no. 8, pp. 997-1007, 2013.

[27] R. Caponetto, G. Dongola, L. Fortuna, and I. Petras, "Fractional order systems: modeling and control applications," World Scientific Series on Nonlinear Science, vol. 72, 2010.

[28] Y. Xinyi, F. Yang, O. Linlin, W. Qunhong, and Z. Weidong, "General stabilization method of fractional-order $P I^{\lambda} D^{\mu}$ controller for fractional-order systems with time delay," International Journal of Robust and Nonlinear Control, vol. 28, no. 16, pp. 4999-5018, 2018.

[29] S. Hafsi, K. Laabidi, and R. Farkh, "A new tuning method for stabilization time delay systems using $P I^{\lambda} D^{\mu}$ controllers," Asian Journal of Control, vol. 17, no. 3, pp. 1-11, 2015.

[30] N. Tan, Ö. Faruk Özgüven, and M. Mine Özyetkin, "Robust stability analysis of fractional order interval polynomials," ISA Transactions, vol. 48, no. 2, pp. 166-172, 2009.

[31] K. Akbari Moornani and M. Haeri, "On robust stability of LTI fractional-order delay systems of retarded and neutral type," Automatica, vol. 46, no. 2, pp. 362-368, 2010. 